\title{
Retinoblastoma: Ten Years Experience at Kanti Children's Hospital
}

\author{
Kailash Prasad Sah, ${ }^{1}$ Rohit Saiju, ${ }^{2}$ Pankaj Roy, ${ }^{\text {Sonali Kafle }}{ }^{1}$ \\ 'Kanti Children's Hospital, ${ }^{2}$ Tilganga Eye Hospital, Kathmandu, Nepal.
}

\section{ABSTRACT}

Introduction: Retinoblastoma is a malignant tumor of the embryonic neural retina. It is the most common intraocular tumor of childhood. It is a disease of early childhood with $80 \%$ cases diagnosed before the age of 4 years. The median age at diagnosis is 2 years. Bilateral cases are diagnosed earlier (median at 12 months and unilateral median age at 24 months). The incidence of retinoblastoma is approximately 1 in 15000-18000 live births in developed countries. The aim of the research is to study the clinical pathological features, treatment and survival of children with retinoblastoma over ten years.

Methods: A retrospective hospital based study was conducted from March 1998 to February 2008. Gender, age, clinical presentation, pathology reports, treatment, follow -up and outcome were recorded at time of diagnosis.

Results: A total of 42 children with retinoblastoma (85.7\% unilateral, 14.3\% bilateral), with 50\% having optic nerve involvement were studied. The most common presenting signs were extraoccular $(28.6 \%)$, proptosis $(23.8 \%)$, leukoria $(23.8 \%)$, phthisis bulbi $(16.7 \%)$, and strabismus $(7.1 \%)$. The age at presentation ranged from 6-120 months with mean age of 46.6 months. The male to female ratio was 1.1:1.Two-thirds presented between 2-5 yrs, followed by 12-24 months (23.8\%). Majority of children had poorly differentiated retinoblastoma (62\%), followed by well differentiated (28.6\%), and moderately differentiated (9.5\%). CEV based protocol used to treat, and over-all 10 yrs survival was $23.8 \%$, death $19 \%$, and lost to follow-up or left against medical advice $57.2 \%$.

Conclusions: Despite severe resource limitation, pediatric oncology unit has been successfully treating retinoblastoma with the success rate of $23.8 \%$.

Keywords: chemotherapy; outcome; remission; retinoblastoma.

\section{INTRODUCTION}

Retinoblastoma is the most common primary ocular malignancy of childhood. ${ }^{1}$ It is a tumor of the embryonic neural retina. Retinoblastoma occurs at a rate of 3.7 cases/million in the United States. There is no racial or sex predilection. Most cases involve only one eye (unilateral), but both eyes may be involved (bilateral) and it may be sporadic or hereditary. ${ }^{2}$ About $60 \%$ cases are unilateral and nonhereditary, $15 \%$ are unilateral and hereditary and $25 \%$ cases are bilateral and hereditary. ${ }^{3}$

Retinoblasts develop from a single cell during the early development of an infant in the womb. ${ }^{4}$ During gestation and early life, these cells are able to divide and multiply. This is the process that helps make enough cells to populate the retina. As children age, their cells undergo a process called differentiation and become mature rods and cones. The cells are no longer able to divide

Correspondence: Dr. Kailash Prasad Sah, Oncology Unit, Kanti Children's Hospital, Kathmandu, Nepal. Email: sahkailash@hotmail.com, Phone: +977-9841265963. 
and multiply, which is why retinoblastoma occurs very rarely after the age of 5 years. Children may be born with retinoblastoma, but the disease is rarely diagnosed at birth. ${ }^{5}$

When retinoblastoma affects both eyes, it is considered a genetic condition. ${ }^{6}$ Rarely, the genetic form occurs only in one eye. The genetic form of the disease occurs in the youngest children (rarely beyond 1 year old) and increases the child's risk of developing another cancer later in life. The risk of additional tumors is higher in children who receive radiation therapy to the orbit to preserve vision or to other parts of the body where the tumor has spread. ${ }^{5}$

\section{METHODS}

A retrospective hospital based study was conducted at Kanti Children's Hospital at the Oncology Unit from March 1998 to February 2008. This hospital is a 500 bedded tertiary level government hospital and the oncology unit has 20 indoor beds, 8 day care beds and outpatient clinics. It has $100 \%$ bed occupancy throughout the year.

A total of 42 post-operative enucleated eye referred cases from Tilganga eye hospital and B.P.K.Ophthalamic centre and research hospital TUTH, Kathmandu Nepal with histo-pathologically retinoblastoma diagnosed children below the age of 10 years were included in the study. All children underwent baseline investigations like full blood count, blood grouping, liver functions, renal functions and electrolytes. Reports of CT-scans of the brain and orbit and chest $\mathrm{x}$-rays were reviewed before the initiation of chemotherapy. Bone scans, cerebrospinal fluid evaluations, and bone marrow evaluationswere only performed when indicated by other clinical, laboratory or imaging studies.

In this study, a CEV (Carboplatin, Etoposide and Vincristine) based protocol was used to treat the children.

After 2 to 4 cycles of chemotherapy, there was ocular examination under anesthesia at the eye hospital. This was also regularly performed during the follow-up period.

The following parameters were recorded in this study: gender, age at diagnosis, clinical presentation, pathology reports, treatment, follow up and outcome.

\section{RESULTS}

Over a ten year period, 42 post-operative children with retinoblastoma were included in this study. The age of the children at presentation ranged from 6 months to 12 months with a mean age of 46.6 months. The male to female ratio was $1.1: 1$ respectively. It is observed that the majority of affected children were in between 2 to 5 years of age i.e. 26 ( $62 \%$ ) followed by children that were in between 1 to 2 years i.e. 10 (23.8\%).

The most common presenting signs were extraocular12 (28.6\%), proptosis $10(23.8 \%)$, leukocoria 10 (23.8\%), phthisis bulbi $7(16.7 \%)$ and strabismus $3(7.1 \%)$. The majority of children had poorly differentiated retinoblastoma 26 (62\%) followed by well differentiated $12(28.5 \%)$ and moderately differentiated 4 (only $9.5 \%)$. Optic nerve involved with malignant cells was present in $21(50 \%)$ of the cases, which means that $50 \%$ was free from infiltration.

Unilateral presentation was present in 36( $85.7 \%)$, followed by bilateral in $6(14.3 \%)$. The overall 10 year survival wasin $10(23.8 \%)$. Death occurred in $8(19 \%)$ cases, and 24 (57.2\%) patients were lost to follow-up or left against medical advice.

\section{DISCUSSION}

Retinoblastoma is the most common intraocular malignancy. ${ }^{7}$ It accounts for about $3 \%$ of all pediatric malignancies. Early diagnosis and multidisciplinary approach and management have led to a gradual improvement in the survival of patients with retinoblastoma over the previous years.

In our study, 36 (85.8\%) of the patients were diagnosed before the age of 5 years (Figure 1). This is slightly lower compared to a study done by Ozkan in Turkey, where the authors found that $92.2 \%$ of the patients were below the age of 4 years. ${ }^{8}$ Another study performed in Turkey, by Ozdemir et al. found that $93.4 \%$ of the patients were diagnosed before the age of 5 years. ${ }^{9}$ Reasons for the fact that the age of presentation is higher in our study are late detection due to delayed medical consultation, parents' ignorance and delayed diagnoses attributed to the lack of skilled manpower. Though it is not common for retinoblastoma, Shrestha et al. described a case of a 37 year old man with retinoblastoma in Nepal. ${ }^{10}$ 


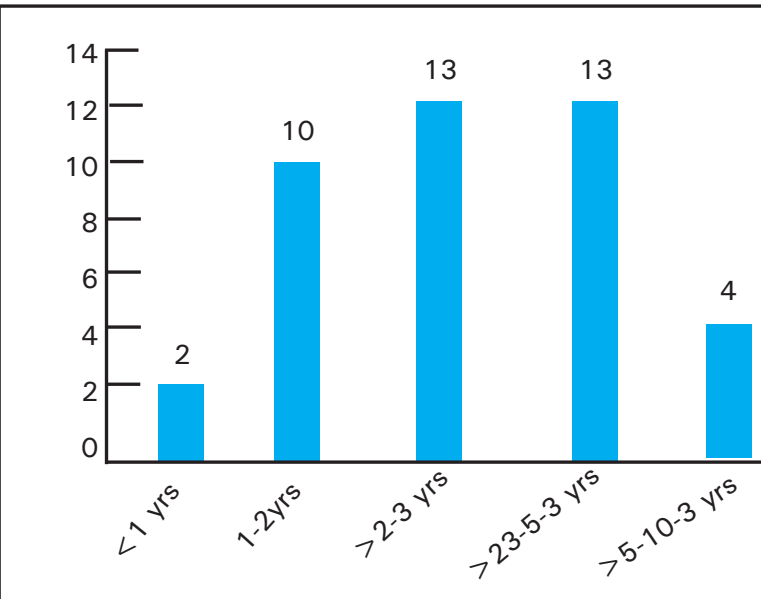

Figure 1. Showing age distribution of retinoblastoma $(n=42)$.

The male: female ratio in this study was 1.1:1 (Figure 2). Similar results were found by Ozkan and Ozdemir, however Naseripour et al. from Iran found this ratio to be slightly higher with $1.4: 1.8 .^{9,11}$

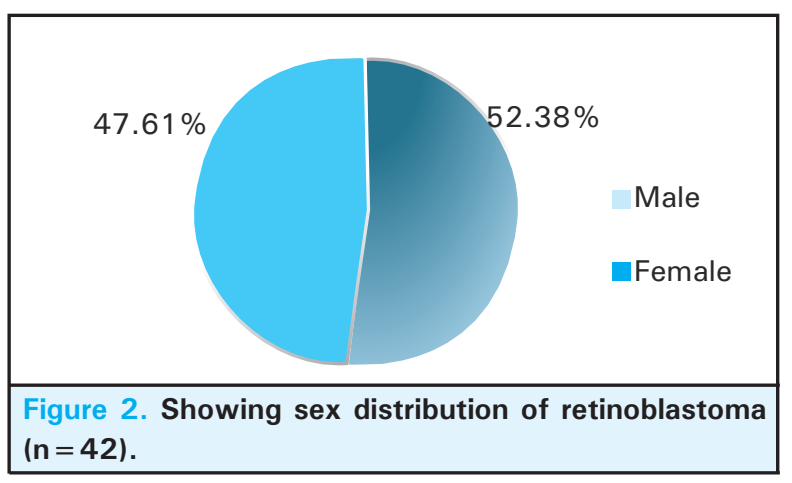

Extraocular presentation was the most common symptom among patients in our hospital 12 (28.6\%), followed by proptosis10 (23.8\%) and leukocoria10( $23.8 \%)$. In contrast, other studies showed that leukocoria is the main presenting symptom with percentages ranging from $72.0 \%$ to $82.3 \%$, followed by strabismus with percentages ranging from $9.9 \%$ to $12.2 \% .^{8,11,12}$ Another studyby Badhu et al. in the eastern part of Nepal showed results similar to ours with proptosis with orbital extension in $40.42 \%$ of the cases, followed by leukocoria in $29.78 \%$ of the cases. ${ }^{7}$ The difference in presenting symptoms may be attributable to late presentation or delayed diagnosis.

In this study we found unilateral involvement in $36(85.7 \%)$ patients and bilateral involvement in 6 $(14.3 \%)$ (Figure 3$)$. Again, similar results were found in the study perfomed by Badhu et al. where unilateral involvement was found in $90.7 \%$ of the cases and bilateral involvement in 9.3\%.7 However, Ozkan, Ozdemir and Naseripour found higher percentages of bilateral involvement, up to $36.7 \% .^{8,9,11}$ This may be due to genetic variations from country to country.

\begin{tabular}{|l|l|}
\hline $50 \%$ \\
\hline
\end{tabular}

Enucleation was performed in all of our cases, while Ozkan, Ozdemir and Naseripour showed lower percentages ranging from $59.3 \%$ to $83 \% .^{8,9,11}$ Late presentation resulted in enucleation in all of our patients. Histopathology shows optical nerve involvement with malignant cells in 21 (50\%) cases (Figure 4). The most common histo-pathological type of retinoblastoma was the poorly differentiated type 26 (61.9\%), followed by well differentiated $12(28.5 \%)$ and moderately differentiated $4(9.52 \%)$ (Table 1$)$.

\begin{tabular}{|c|c|c|}
\hline & Type & n (\%) \\
\hline 1. & Well differentiated & $12(28.5 \%)$ \\
\hline 2. & Moderately differentiated & $4(9.52 \%)$ \\
\hline 3. & Poorly differentiated & $26(61.9 \%)$ \\
\hline & tal number & 42 \\
\hline
\end{tabular}

\begin{tabular}{|l|l|}
\hline \\
\hline
\end{tabular}




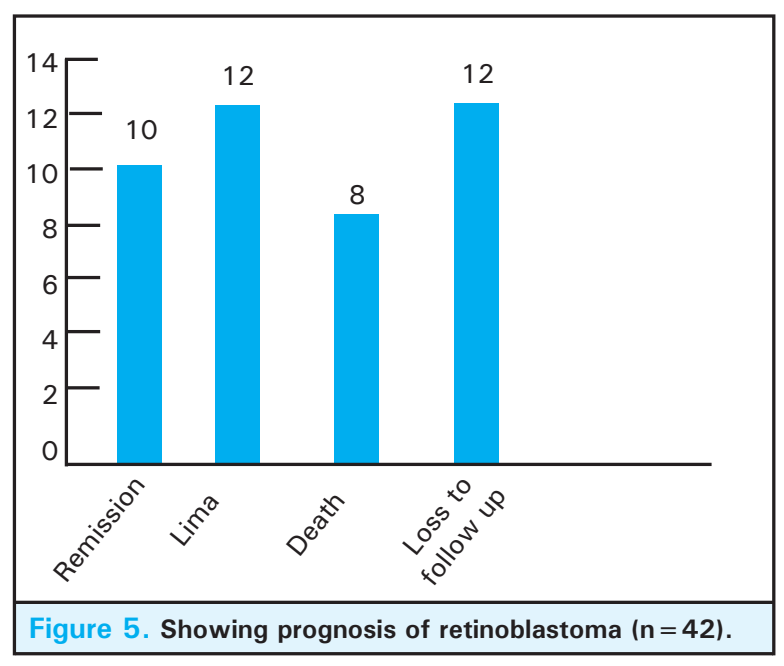

The 10 years survival rate in this study was $10(23.8 \%)$ (Figure 5). Comparing this result with developed countries like Iran and the USA, we have found poorer outcomes. Naseripour found the 10 year survival rate in Iran 11 to be $69.2 \%$ and Broaddus found this to be
92.9\% in the USA. ${ }^{13}$ This may be due to early detection and early treatment in more developed countries. In this study $24(57.2 \%)$ of our patients were lost to follow up which is primarily attributed to the financial burden, high costs of chemotherapy, poor socio-economic condition of the parents and lack of accommodation.

\section{CONCLUSIONS}

This study showed the current state of affairs of treatment of retinoblastoma in Nepal. We found 10 year survival rates to be lower than in more developed countries, most likely due to delayed presentation and treatment. If retinoblastoma could be diagnosed in its early stage, less aggressive treatment modalities would be possible and there is a better chance of preservation of the eye and survival rates will be higher.

\section{ACKNOWLEDGEMENTS}

We would like to thank the entire oncology unit staffs, parents, children and doctors. Special thanks to Anne Verheijen and Sharon Vrooijink for their help.

\section{REFERENCES}

1. Albert DM. Historic review of retinoblastoma Ophthalmology. 1987;94:654-62.

2. Bishop JO, Madson EC. Retinoblastoma: review of the current status. SurvOphthalmol. 975;19:342-66.

3. Shields JA, Augsburger JJ. Current approaches to the diagnosis and management of retinoblastoma. SurvOphthalmol. 1981;25:347-72.

4. Murphree LA. Molecular genetics of retinoblastoma. OphthalmolClin N Am. 1995;8:155-66.

5. Knudson AG Jr. Mutation and cancer: statistical study of retinoblastoma. ProcNatlAcadSci U S A. 1971;68:820-3.

6. Tamboli A, Podgor MJ, Horm JW. The incidence of retinoblastoma in the United States: 1974 through 1985. Arch Ophthalmol. 1990;108:128-32.

7. Badhu B, Sah SP, Thakur SK et al. Clinical presentation of retinoblastoma in Eastern Nepal. Clin Experiment Ophthalmol. 2005 Aug;33(4):386-9.
8. Ozkan A1, Pazarli H, Celkan T, Karaman S, Apak H, Kaner G,et.al. Retinoblastoma in Turkey: survival and clinical characteristics 1981-2004. Pediatr Int. 2006 Aug;48(4):369-73.

9. Ozdemir H, Tacyildiz N, Unal E, Yavuz G, Ugur H, Gunduz K. Clinical and epidemiological characteristics of retinoblastoma: correlation with prognosis in a Turkish pediatric oncology center. PediatrHematolOncol. 2007 Apr-May;24(3):221-31.

10. Shresta A, Adhikari RC, Saiju R. Retinoblastoma in a 37 years old man in Nepal: a case report. Kathmandu University Medical Journal. 2010;8(30):247-50.

11. Naseripour M, Falavarjani K, Bakhtiari P, etal. Retinoblastoma survival in Iran: 10 years experience of a referral center. Iranian Journal of Ophthalmology.2009;21:17-24.

12. Shuwei B, Ruojin R, Bin L, et al. Delay in the diagnosis of retinoblastoma in China. Actaphthalmica. 2011;89:e72-4.

13. Broaddus E, Topham A, Singh AD. Survival with retinoblastoma in the USA: 1975-2004. Br J Ophthalmol 2009;93: 24-7. 